\title{
IoT Based Fire Accident Detection System with Deep Learning Intelligence
}

\author{
Hitanshi Jain, Sai Teja Miyapuram, Sree Ranga Reddy Bobbala
}

\begin{abstract}
A fire accident can be caused by many hazards, such as a propane tank, a defective product, a vehicle crash, or poor workplace safety. Because accidents involving fire are often unexpected and sudden, there isn't a standard legal process for dealing with them, other than filing a negligence or workers compensation claim. This project aims to detect and monitor Fire Accident incidents well in advance and alert the surroundings to minimize the losses. This is an integration of IoT and Deep Learning Technologies, where sensors are used to collect the relevant data under the supervision of a controller unit. The controller unit collects and sends this data to a cloud database, from where the data for the Deep Learning model is fetched. This data is then used for making some insights and further predictive analytics. From the insights, many variables were found to be one of the reasons for a fire accident to take place. We considered the information about variables like Flame sensor, Temperature, Heat Index, GPS coordinates, Smoke, Type of Gases, Date, and Time for feature set generation and fed the model to a deep neural network for making future predictions. Comparing to existing conventional methods, this proposed method is different in terms of integrating deep learning with IoT. This method of approach will predict the chance of accidents priorly by classification of data.
\end{abstract}

Key Terms: Deep Learning, IoT, Data Analytics, Cloud Database, Sensors

\section{INTRODUCTION}

In the process of managing a fire accident place, it is laborious and arduous to analyze the situation. As technology is increasing vastly, industries are also increasing proportionally. The main problem here is to maintain fire safety at every moment. If we recollect the major fire accidents in the year 2020, there are many which include home fires, electrical fires, catastrophic multipledeath fires, fireworks fires, etc. As part of this paper, let us see the Beirut port explosion on August 4, 2020. Beirut's port explosion [1] is one of the non-nuclear explosions which affected the crunching of the port and destroyed about half the city. The main cause of this incident is the detonation of 2,700 tons of ammonium nitrate, which is a combustible chemical compound used to manufacture explosives.

Manuscript received on October 03, 2021.

Revised Manuscript received on October 10, 2021.

Manuscript published on October 30, 2021.

* Correspondence Author

Hitanshi Jain, B.Tech., Department of Mechanical Engineering, Indian Institute of Technology (BHU) Varanasi, India. Email: hitanshijain27@gmail.com

Sai Teja Miyapuram*, B.Tech. Department of Electronics and Communication Engineering, SR Engineering College, Warangal, India. Email: saitejamiyapuram15@gmail.com

Sree Ranga Reddy, Department of Computer Science, Cleveland State University, OH, USA. Email: bobbalaranadheerreddy@gmail.com

(C) The Authors. Published by Blue Eyes Intelligence Engineering and Sciences Publication (BEIESP). This is an open access article under the CC BY-NC-ND license (http://creativecommons.org/licenses/by-nc-nd/4.0/)
This explosion killed 218 people, wounded 7000 people, displacing over 300,000 people, and impacted 56\% of private businesses in Beirut. From the World Bank Analytics, the explosion made $\$ 4$ billion in material damage. In Fig. 1., there are many causes involving in the Beirut incident. Like this, there are many fire accidents and explosions globally, like Australia Bush Fire, Amazon. Forest Fire, and many more at a low level inside every nation. The proposed method given in this paper is to predict the probability of a fire accident occurring at any place and alert the situation to reduce the losses. Sensors and microcontroller units will work in collaboration to collect the conditional data. Once the required data is collected, it is sent to a cloud database over the internet within a short period. The data is fed to a deep learning model to make predictions. This method of implementation in fire safety will monitor fire accident-prone areas without human involvement, predict future instances, and reduces losses.

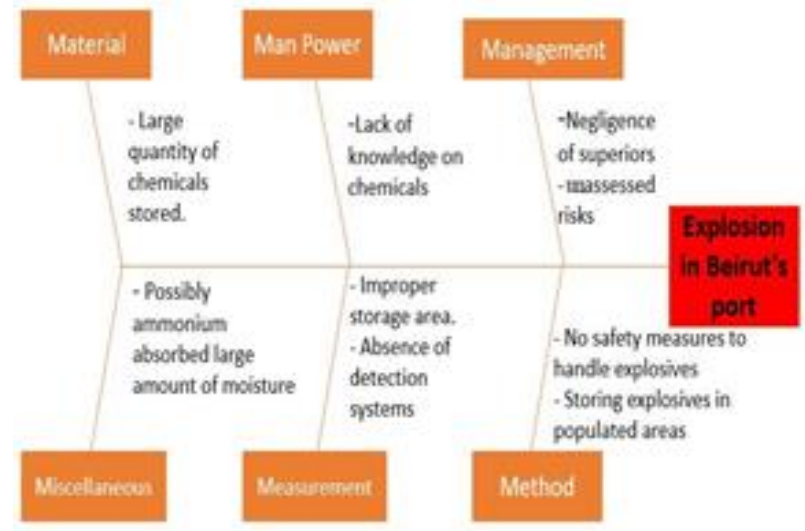

Fig. 1. Investigative Consequence Analysis of Beirut's Port Explosion.

\section{MATERIALS AND METHODS}

\section{A. Sensors with Microcontroller unit}

In this method, sensors like the Infrared Flame sensor are used to detect the fire, it has high photosensitivity, wide directional angle, and fast response time. DHT11 is used to monitor the temperature, humidity and to estimate the heat index. GPS module is integrated to trace the location details of the accident and to analyze the affected area. A gas sensor is used to measure gases like LPG, CO, $\mathrm{CH}_{4}$, Alcohol, Propane, Smoke in the surrounding area, which are the major causes in fire accident zones. DHT11 is commonly used to measure the temperature and humidity of any area, from those we calculate the heat index. The heat index has a crucial role in a fire accident zone, which will convey the severity of the accident. This sensor works on a $3.5 \mathrm{~V}$ supply voltage and has an accuracy of $\pm 1^{\circ} \mathrm{C}$.

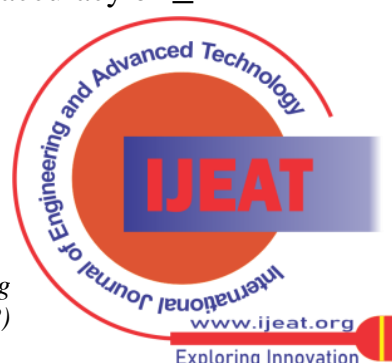
and Sciences Publication (BEIESP) (C) Copyright: All rights reserved. 


\section{IoT Based Fire Accident Detection System with Deep Learning Intelligence}

Neo 6 is a GPS module that is used to get the location details, it gives both longitude and latitude numbers with precise point positioning. A gas sensor MQ-2 is used to measure the levels of various gases in ppm.

All these sensors are connected to Node MCU, a microcontroller unit, which will receive and process the data. Node MCU can connect to the internet over Wi-Fi and can send the data to cloud databases. In the cloud, database data is organized into a table. Then organized data is exported to make predictive analytics [2].

\section{B. Characterization of gases with gas sensor}

In Fig. 2., the MQ-2 gas sensor can detect and measure $\mathrm{H}_{2}$, LPG, CO, $\mathrm{CH}_{4}$, Alcohol, Smoke levels in ppm, that depends on the $R_{s} / R_{0}$ ratio, where Rs is the sensor resistance at various concentrations of gases and $R_{0}$ is the sensor resistance at $1000 \mathrm{ppm}$ of $\mathrm{H}_{2}$ in the clean air. From the chart, $\mathrm{R}_{\mathrm{S}} / \mathrm{R}_{\mathrm{o}}$ ratio is inversely proportional to the concentration of gases in ppm. Based on this ratio levels of various gases are measured in ppm. If high range ppm is obtained for any of the gases, that gas may be the actual cause of the fire accident. And in some fire accidents smoke may be the first effect seen before the accident occurs, so depending on limitations of smoke levels, chances of fire accident are also predicted in the deep neural network model [3].

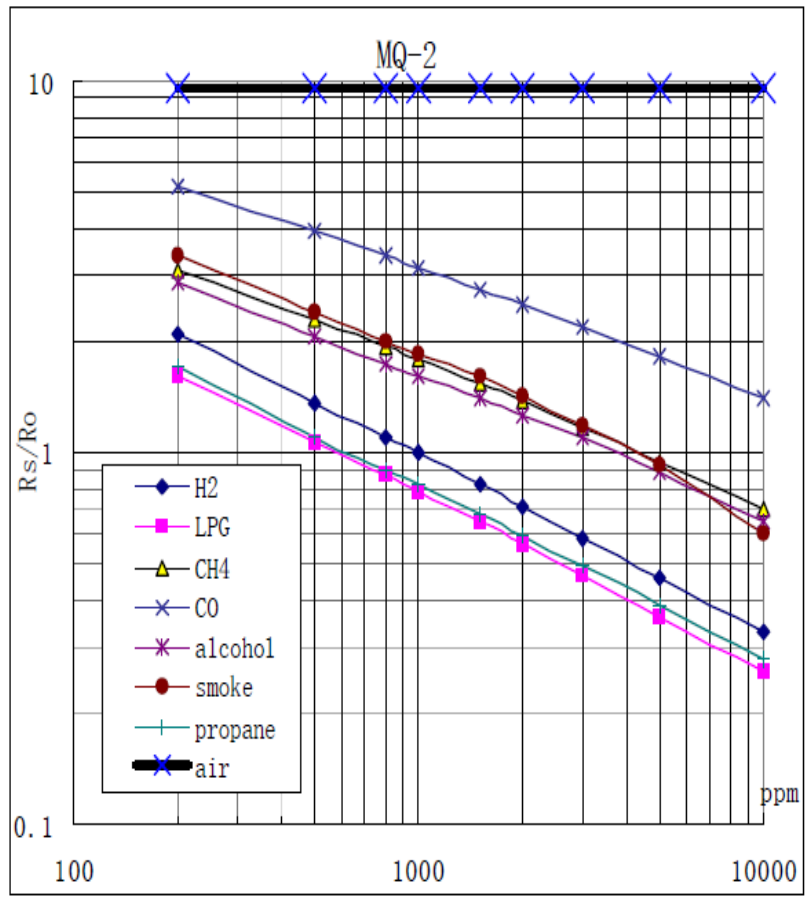

Fig. 2. Sensitivity characteristics of MQ-2 gas sensor.

\section{Calculation of Heat Index}

Heat Index is calculated using the formula [4]:

$$
\begin{aligned}
H I= & C_{1}+C_{2} T+C_{3} R+C_{4} T R+C_{5} T^{2}+C_{6} R^{2}+ \\
& C_{5} T^{2} R+C_{8} T R^{2}+C_{9} T^{2} R^{2}
\end{aligned}
$$

Where, $\mathrm{HI}=$ Heat Index, $\mathrm{T}=$ ambient temperature in ${ }^{\circ} \mathrm{C}, \mathrm{R}=$ relative humidity, and $\mathrm{C}_{1}$ to $\mathrm{C}_{9}$ are constants. $\mathrm{C}_{1}=$ 8.784694755, $\mathrm{C}_{2}=1.61139411, \mathrm{C}_{3}=2.3385488388$,

$\mathrm{C}_{4}=-0.14611605, \quad \mathrm{C}_{5}=-0.01230809, \quad \mathrm{C}_{6}=-$ $0.0164248277, \mathrm{C}_{7}=0.002211732, \mathrm{C}_{8}=0.00072546, \mathrm{C}_{9}=-$ 0.000003582 . These constants are only when temperature is in Celsius.

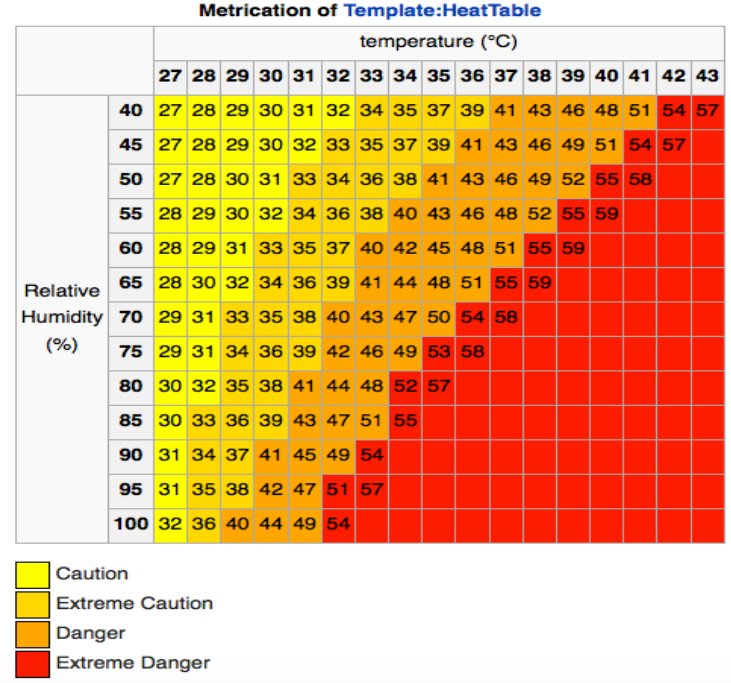

Fig. 3. Heat Index Chart

After, calculating the heat index values using temperature and humidity using the DHT11 sensor [5], the severity of the fire accident is analyzed using the heat index chart given in Fig. 3. From the chart, high index values are lying in extreme danger and dangerous parts, this will help us to analyze the severity of the occurred incident. Meanwhile, caution and extreme caution values will help us to predict the incident priorly and alert the situation. Heat index plays a key role in the deep learning model to make decisions accurately.

\section{Data Analytics and Deep Learning Model}

From the cloud database, the data is fetched as a data frame which is having Flame, Temperature, Humidity, Heat Index, Longitude, Latitude, Date, Time, CO Gas, LPG Gas, Smoke as attributes. By performing EDA on the dataset, the following insights are the draw. In Fig. 4., plots depict the data distribution comparing one variable with another, based on the accident status. Firstly, flame and smoke are detected and it is very clear that Heat Index, Temperature, and levels of one or more gases are high when there is a possibility of a fire accident. Conversely, these values are low when there is no chance of a fire accident. Also, either flame or smoke is detected in the fire accident zone, or maybe both.

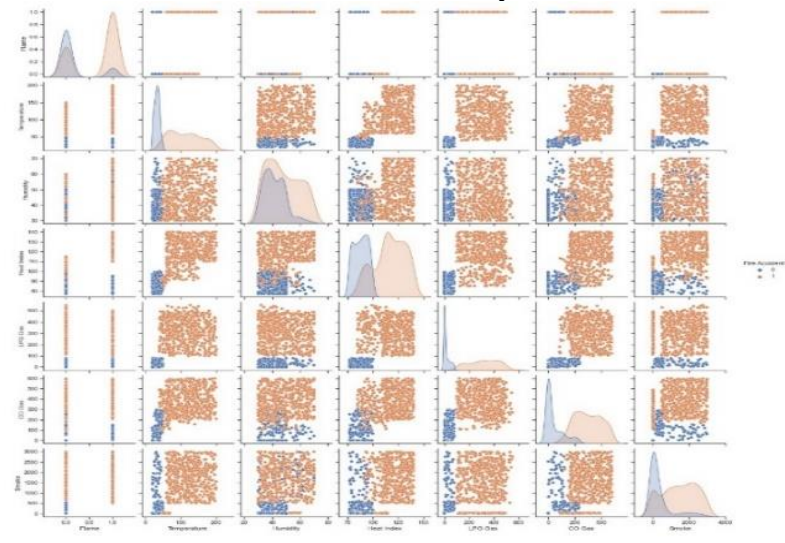

Fig. 4. Plots of all available variables based on accident status.

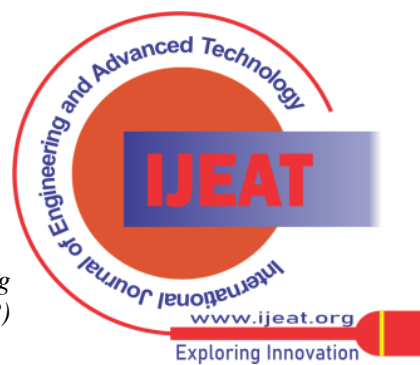


A three-layered DNN built using TensorFlow and Keras is used to classify the chances of fire accident and has an accuracy of $94 \%$ with an epoch value of 10 . Activation of the sigmoid is used to classify the data, whether a fire accident is going to happen or not. And the predicted probabilities are also available to monitor the situation.

\section{PROPOSED SYSTEM}

\section{A. Block Diagram}

Fire accidents are one of the disastrous incidents that will occur and make huge losses. Predicting this well in advance will save many lives and properties The major indicators of fire accidents are Flame, Heat Index, Temperature, Various types of gases. This paper discusses the pilot project of employing a deep learning model associated with IoT, to monitor and predict the situation at any place.

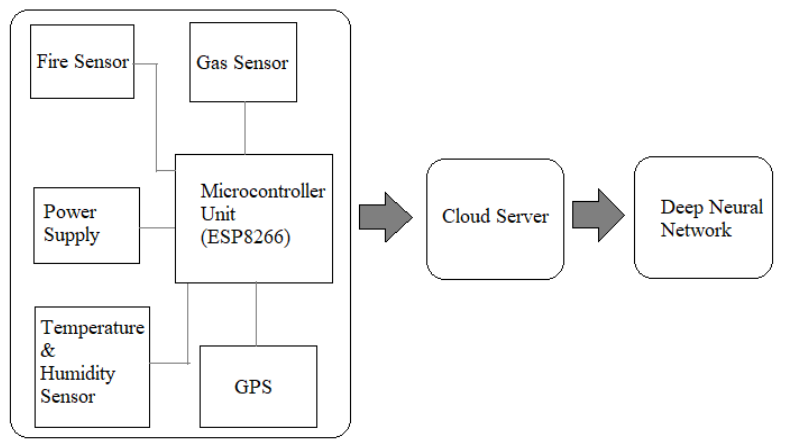

Fig. 5. Block diagram of the proposed system.

The system consists of Node MCU, along with temperature humidity sensor, flame sensor, gas sensor, GPS module as shown in Fig. 4. All the data is collected continuously by all the sensors, Node MCU takes control over all the sensors and processes the organized data. This organized data is sent to a real-time cloud database. After data is received into the database, data is organized into a table. From the cloud database, data can be accessed anywhere in the world. Data from the cloud is imported into the host machine to perform data analytics and implement a deep learning model to predict future events.

\section{B. Flow Chart}

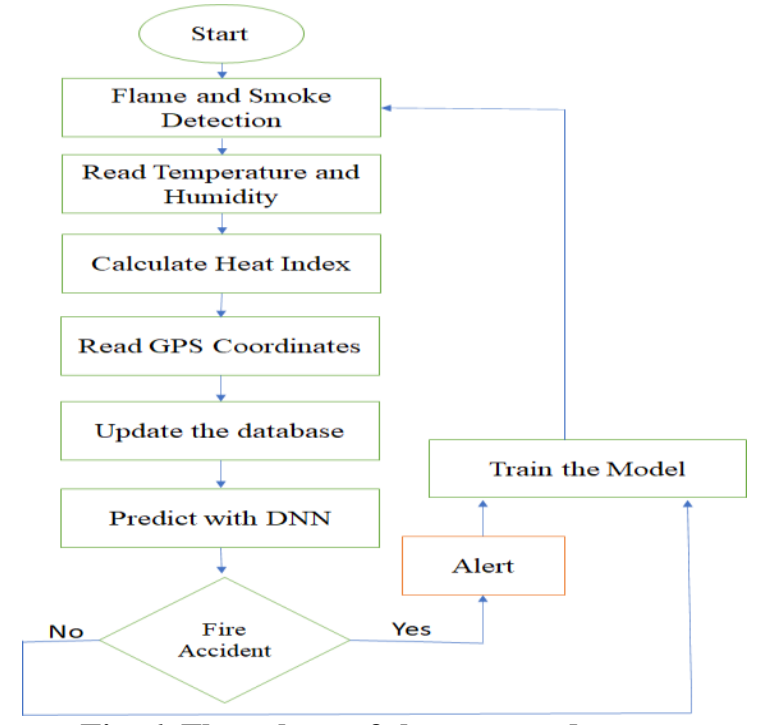

Fig. 6. Flow chart of the proposed system
The above-given flow chart explains the sequence of steps involved in this project. This process is so simple to detect, monitor, and predict the situation. Firstly, a Flame sensor is always ready to detect the fire, simultaneously, the Gas Sensor detects the availability of smoke in the surroundings. If the fire and smoke levels are within the limits. Then, using the temperature sensor, temperature and humidity is measured, which is used to evaluate the heat index. The GPS coordinates longitude and latitude numbers are noted to see locate the source of explosion or fire. Every time the data is stored in a cloud database to make real-time monitoring. A pre-trained DNN is used to predict the chances of an accident. This model always learns the new instance after each iteration, whether a fire accident occurred or not, it improves the accuracy of the model. This kind of implementation may save many dangers by predicting future events based on various factors. Since the model is always in encounter training after each iteration, it will learn the new instances and predict with more accuracy.

\section{IMPLEMENTATIONS AND RESULTS}

\section{A. IoT Device}

The figure in Fig. 5. is the prototype stage of the project in this paper, which is used to collect the required data. All the sensors are connected to a microcontroller unit and capsuled in a single unit kit [6]. A power supply is provided within the kit and it automatically connects to the internet through Wi-Fi. This kit includes a DHT11 temperature sensor, Flame sensor, MQ-2 gas sensor, buzzer, Node MCU, GPS module, and power supply unit.

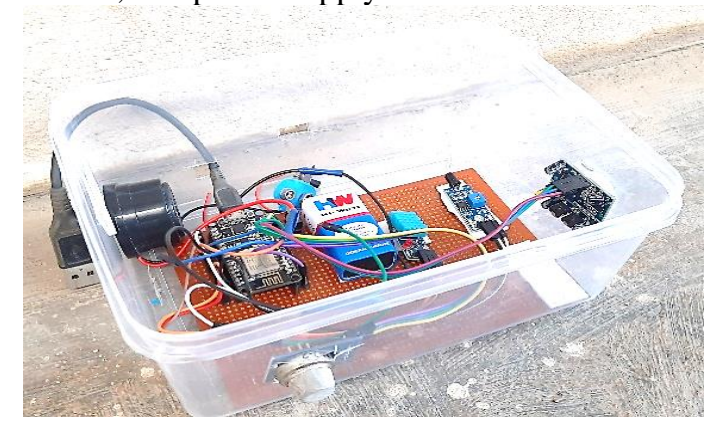

Fig. 7. IoT Device.

\section{B. Cloud DataBase}

The cloud database for this project is Google Firebase Realtime Database. All the sensor data is placed in a structured manner. Using Firebase support libraries in Arduino IDE, the structure is implemented.

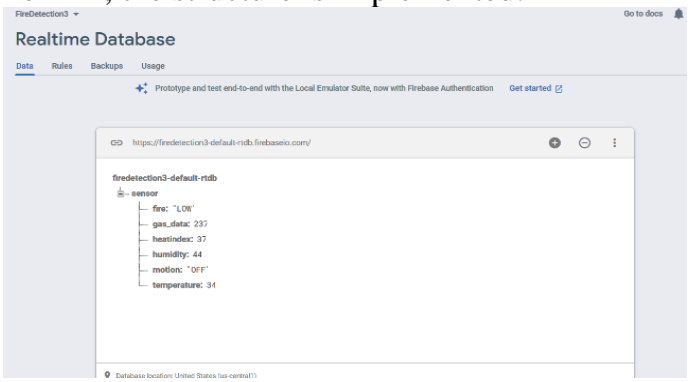

Fig. 8. Google Firebase Realtime Database.

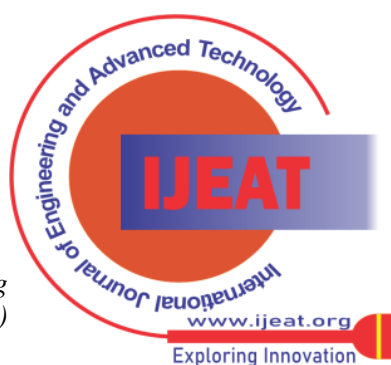




\section{IoT Based Fire Accident Detection System with Deep Learning Intelligence}

In Fig. 6, the data is organized into various fields from various sensors. The data in the firebase is continuously updating and stores the history of previous values. Data is imported to the host machine using API calling in JSON format. This data is now used to make some data analytics and build the DNN model.

\section{DNN Model}

A DNN is an artificial neural network with multiple layers, layers consist- The input layer, output layer, and many hidden layers. This system uses its layers of nodes to make high-level functions from input information. The model summary of implemented Deep learning model is given below. It is a three-layered neural network to classify the data. The first two layers use ReLU activation and the final layer uses sigmoid activation to transform values between 0 to 1 [7]. The model will accept 8 inputs and give output as one class label. This simple deep learning model is foretelling the upcoming fire accidents.

Model: "sequential"

\begin{tabular}{llc}
\hline Layer (type) & Output Shape & Param \# \\
\hline \hline dense (Dense) & (None, 8) & 64 \\
\hline dense_1 (Dense) & (None, 4) & 36 \\
\hline dense_2 (Dense) & (None, 1) & 5 \\
\hline
\end{tabular}

Total params: 105

Trainable params: 105

Non-trainable params: 0

Fig. 9. Model Summary.

\section{E. Results}

The implemented DNN has had an accuracy of $94 \%$ and can measure the probability of fire accidents happening. IoT device is also able to send the data to cloud with no loss of time. As the data is fetched to DNN it is predicting the chance of fire accident. As the model is always learning on the new data; its accuracy is appreciable. Even if DNN predicts wrongly, the IoT device is equipped with a buzzer to give the alarm. All these implementations aggregately are supportive to prevent a fire accident and protect lives.

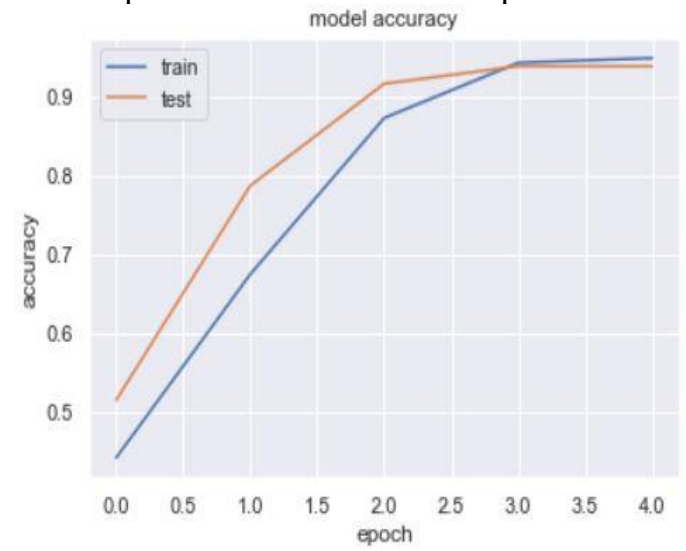

Fig. 10. Model accuracy

We split data in the ratio of 80:20 for the initial training of the model, however, a new training instance is created after every iteration of the flowchart. The accuracy obtained is
$94 \%$ and we can visualize the test and train accuracy in the above figure fig. 9.

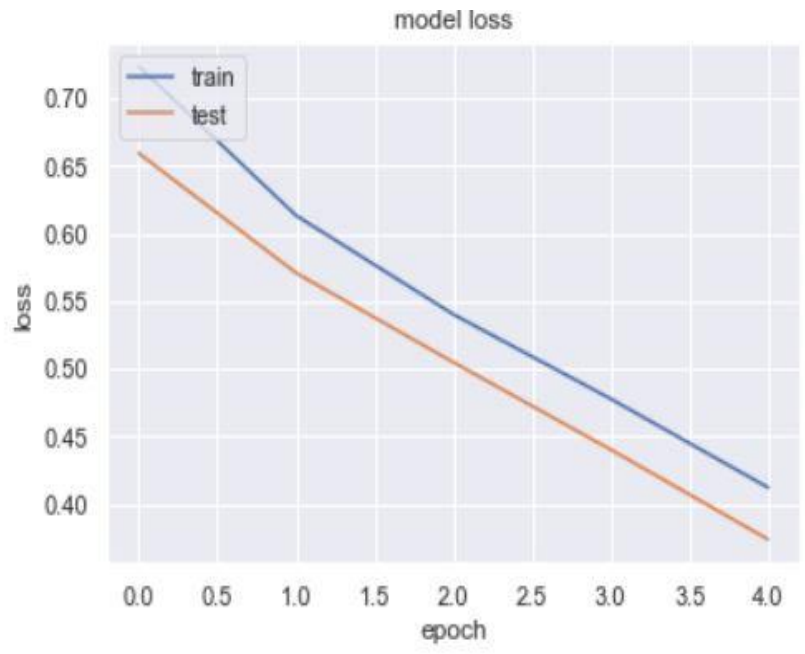

Fig. 11. Model loss.

\section{CONCLUSION AND FUTURE WORK}

IoT and Deep Learning are now giving rise to AIOT, with this support, it is very unique and easy to avoid a fire accident. Using the proposed solution most of the manual works is reduced. High safety is provided in fire accidentprone areas, which will reduce loss of lives and economy. From the chart in Fig. 8, it is concluded that most of the accident chances are due to the presence of combustible gases. This method of fire safety implementation can predict dangerous fire accidents priorly. Any changes in the temperature, heat index, presence of a flame, smoke, low ignition compound, and gases are monitored without human involvement and accurate decision making to save the economy and ecological system

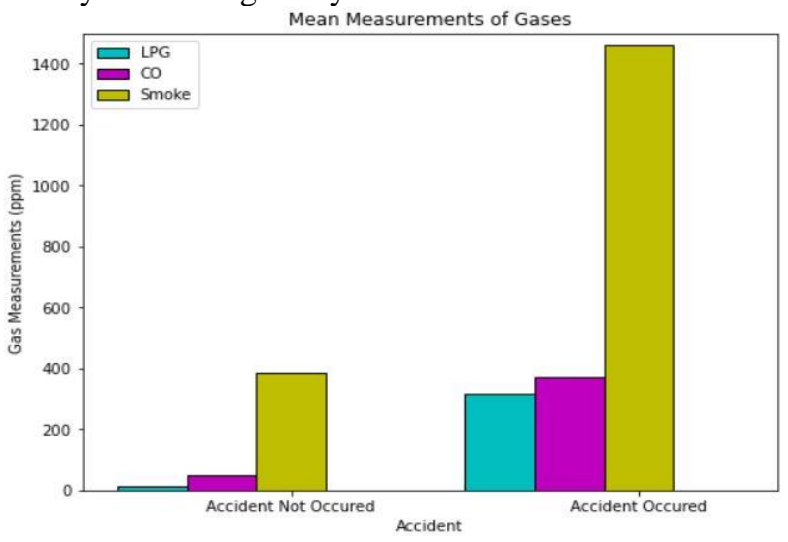

Fig. 12. Gases causing fire accidents.

This paper's scope is limited to measure LPG because it is mostly used for domestics and laboratory uses. And high levels of smoke are available in fire accident cases. However, in the future many features can be added to make the final system [8], i.e., adding more sensors to detect any kind of combustible gases which is safer, efficient, accurate, alarm notification, mobile application-based system, etc.

Published By:

Blue Eyes Intelligence Engineering and Sciences Publication (BEIESP) 


\section{ACKNOWLEDGEMENT}

The authors would like to thank all the reviewers for spending their invaluable time. Thank you.

\section{REFERENCES}

1. Broomandi, P., Jahanbakhshi, A., Nikfal, A. et al. Impact assessment of Beirut explosion on local and regional air quality. Air Qual Atmos Health (2021).

2. Reddy, M. S., \& Rao, K. R. (2016). Fire accident detection and prevention monitoring system using wireless sensor network-enabled android application. Indian Journal of Science and Technology, 9(17), 1-5.

3. Gautam, A., Verma, G., Qamar, S., \& Shekhar, S. (2021). Vehicle pollution monitoring, control, and challan system using MQ2 sensor based on internet of things. Wireless Personal Communications, 116(2), 1071-1085.

4. Rothfusz, L. P., \& Headquarters, N. S. R. (1990). The heat index equation (or, more than you ever wanted to know about heat index). Fort Worth, Texas: National Oceanic and Atmospheric Administration, National Weather Service, Office of Meteorology, 9023.

5. Srivastava, D., Kesarwani, A., \& Dubey, S. (2018). Measurement of Temperature and Humidity by using Arduino Tool and DHT11. International Research Journal of Engineering and Technology (IRJET), 5(12), 876-878.

6. IoT-based Waste Management System and Attendance Monitoring of Workers for Smart Cities Development - M Sai Teja, D Koushik, K Sushma, G Ravi Prasad, K Sreedhar Reddy. International Research Journal of Engineering and Technology (IRJET) Volume 7, Issue 2, February 2020

7. X Waoo, A. A., \& Soni, B. K. (2021). Performance Analysis of Sigmoid and Relu Activation Functions in Deep Neural Network. In Intelligent Systems (pp. 39-52). Springer, Singapore.

8. Lee, D. S., Huh, J. S., \& Lee, D. D. (2003). Classifying combustible gases using a micro-gas sensor array. Sensors and Actuators B: Chemical, 93(1-3), 1-6.

9. Daniel Pires Bitencourt, Paulo Alves Maia, Rodrigo Cauduro Roscani. (2020) The heat exposure risk to outdoor workers in Brazil. Archives of Environmental \& Occupational Health 75:5 pages 281-288.

10. Planas-Cuchi, E., Montiel, H., \& Casal, J. (1997). A survey of the origin, type, and consequences of fire accidents in process plants and the transportation of hazardous materials. Process Safety and Environmental Protection, 75(1), 3-8.

11. Kabir, M. Y., \& Madria, S. (2019, November). A deep learning approach for tweet classification and rescue scheduling for effective disaster management. In Proceedings of the 27th ACM SIGSPATIAL International Conference on Advances in Geographic Information Systems (pp. 269-278).

\section{AUTHORS PROFILE}

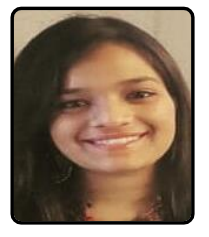

Hitanshi Jain is currently in her final year, pursuing B.Tech. in Mechanical engineering from the Indian Institute of Technology (BHU) Varanasi. She has acquired rigorous training as a summer intern from HCL Technologies, gaining enriching industrial exposure in the software development field. Her interests lie in the fields of Data Mining, Deep Learning, and Software Development. For further exploration, she is working on the research topic "Link Prediction in Dynamic Social Networks" using a combination of similarity indices and training the model with appropriate machine learning algorithms under the guidance of a professor from the Computer Science department of IIT BHU.

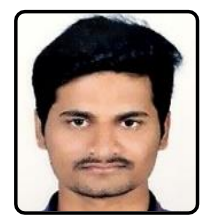

Sai Teja Miyapuram, completed his B.Tech. Electronics and Communication Engineering from SR Engineering College, Warangal, 2016-2020, and been the part of IEEE Student member. Published a paper titled- IoT based Waste Management System and Attendance Monitoring of Workers for Smart Cities Development in IRJET and filed for patent. Been part of National Level Makeathon organized by Indian Electronics and Semiconductor Association, New Delhi. Worked collaboratively with IISc Bangalore alumni on seamless connectivity with Bluetooth Low Energy module. Areas of research interest are IoT, Data Mining, Machine Learning, and Deep Learning.

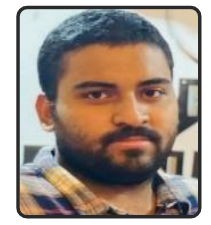

Sree Ranga Reddy Bobbala is currently pursuing his master's in computer science from Cleveland State University, USA. As a part of my master's research, I contribute my knowledge in a research paper. Previously, worked on IoT and Robotic projects at my graduation. Been the part of projects in under graduation and masters. Areas of interest are Data Science, Data Mining, IoT, Software development.
Published By:

Blue Eyes Intelligence Engineering and Sciences Publication (BEIESP) (C) Copyright: All rights reserved.

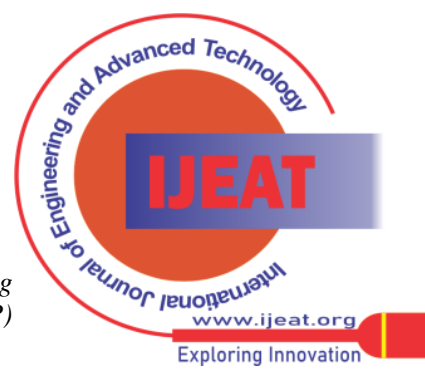

